\title{
CONTROLLABILITY OF FRACTIONAL INTEGRODIFFERENTIAL EQUATIONS WITH STATE-DEPENDENT DELAY
}

\author{
KHALIDA AISSANI AND MOUFFAK BENCHOHRA \\ Communicated by Neville Ford
}

\begin{abstract}
According to fractional calculus theory and Sadovskii's fixed point theorem, we establish sufficient conditions for controllability of the fractional integro-differential equation with state-dependent delay. An example is provided to illustrate the theory.
\end{abstract}

1. Introduction. The purpose of this paper is to establish sufficient conditions for controllability of the fractional integro-differential equation of the form

$$
\begin{aligned}
D_{t}^{q} x(t)= & A x(t)+B u(t) & & \\
& +\int_{0}^{t} a(t, s) f\left(s, x_{\rho\left(s, x_{s}\right)}, x(s)\right) d s, & & t \in J=[0, T], \\
x(t)= & \phi(t), & & t \in(-\infty, 0],
\end{aligned}
$$

where $D_{t}^{q}$ is the Caputo fractional derivative of order $0<q<1, A$ is a generator of an analytic semigroup $\{S(t)\}_{t \geq 0}$ of uniformly bounded linear operators on $X, f: J \times \mathcal{B} \times X \rightarrow X$ and $\rho: J \times \mathcal{B} \rightarrow(-\infty, T]$ are appropriated functions, $a: D \rightarrow \mathbb{R}(D=\{(t, s) \in J \times J: t \geq s\})$, $\phi \in \mathcal{B}$ where $\mathcal{B}$ is called the phase space, to be defined in Section 2. $B$ is a bounded linear operator from $X$ into $X$, the control $u \in L^{2}(J ; X)$, the Banach space of admissible controls. For any function $x$ defined on $(-\infty, T]$ and any $t \in J$, we denote by $x_{t}$ the element of $\mathcal{B}$ defined by

$$
x_{t}(\theta)=x(t+\theta), \quad \theta \in(-\infty, 0] .
$$

2010 AMS Mathematics subject classification. Primary 26A33, 34A08, 34G20, 34H05, 34K37, 35R11, 93B05.

Keywords and phrases. Controllability, Caputo fractional derivative, integrodifferential equations, fixed point, semigroup, state-dependent delay.

Received by the editors on March 19, 2014, and in revised form on November 3, 2015.

DOI: $10.1216 /$ JIE-2016-28-2-149

Copyright (C)2016 Rocky Mountain Mathematics Consortium 
Here $x_{t}$ represents the history of the state up to the present time, $t$.

Fractional differential equations have recently been proved to be valuable tools in the modeling of many phenomena in various fields of science and engineering, so they attracted many researchers (cf., e.g., $[3,32]$ and references therein). On the other hand, integrodifferential equations arise in various applications such as viscoelasticity, heat equations and many other physical phenomena (cf., e.g., $[13,25,26,27,42]$ and references therein). The existence of fractional differential equations with state-dependent delay are one of the theoretical fields that have been investigated by many authors $[\mathbf{2}, \mathbf{1 8}]$. Very recently, Benchohra and Litimein $[\mathbf{1 2}, \mathbf{1 4}]$ have investigated the existence and uniqueness of a mild solution for fractional integral and integro-differential equations with state-dependent delay on infinite interval, whereas Kavitha et al. [23] have studied the existence of mild solutions for neutral functional fractional differential equations with state-dependent delay.

Controllability is one concept of control dynamic systems that some classes of such systems can be represented by nonlinear differential equations $[\mathbf{1}, \mathbf{5}, \mathbf{1 0}, \mathbf{1 5}, \mathbf{1 6}]$. In recent years, the problems of controllability for various kinds of fractional differential and integrodifferential equations have been discussed in $[4,6,11,17,22,24,30,31,38,39]$. Recently, in [37], the authors established sufficient conditions for the approximate controllability of certain classes of abstract fractional evolution equations in Hilbert spaces.

The aim of our paper is to establish controllability results for fractional evolution integrodifferential systems with state-dependent delay by using fractional calculus and Sadovskii's fixed point theorem, combined with the Kuratowski measure of noncompactness. An example is presented to show an application of the abstract results.

2. Preliminaries. In this section, we include some notations, definitions and theorems needed to establish our results.

Let $(X,\|\cdot\|)$ be a real Banach space, $C(J, X)$ the Banach space of all $X$-valued continuous functions on $J$ with norm

$$
\|y\|_{\infty}=\sup \{\|y(t)\|: t \in J\}
$$

$L(X)$ the Banach space of all linear and bounded operators on $X$, 
$L^{1}(J, X)$ the space of $X$-valued Bochner integrable functions on $J$ with the norm

$$
\|y\|_{L^{1}}=\int_{0}^{T}\|y(t)\| d t .
$$

$L^{\infty}(J, \mathbb{R})$ is the Banach space of essentially bounded functions, normed by

$$
\|y\|_{L^{\infty}}=\inf \{d>0:|y(t)| \leq d, \text { almost everywhere } t \in J\} .
$$

In this paper, we will employ an axiomatic definition for the phase space $\mathcal{B}$ which is similar to that introduced by Hale and Kato [19]. Specifically, $\mathcal{B}$ will be a linear space of functions mapping $(-\infty, 0]$ into $X$ endowed with a seminorm $\|\cdot\|_{\mathcal{B}}$ and satisfies the following axioms:

(A1) If $x:(-\infty, T] \rightarrow X$ is continuous on $J$ and $x_{0} \in \mathcal{B}$, then $x_{t} \in \mathcal{B}$ and $x_{t}$ is continuous in $t \in J$ and

$$
\|x(t)\| \leq C\left\|x_{t}\right\|_{\mathcal{B}},
$$

where $C \geq 0$ is a constant.

(A2) There exist a continuous function $C_{1}(t)>0$ and a locally bounded function $C_{2}(t) \geq 0$ in $t \geq 0$ such that

$$
\left\|x_{t}\right\|_{\mathcal{B}} \leq C_{1}(t) \sup _{s \in[0, t]}\|x(s)\|+C_{2}(t)\left\|x_{0}\right\|_{\mathcal{B}},
$$

for $t \in[0, T]$ and $x$ as in (A1).

(A3) The space $\mathcal{B}$ is complete.

Remark 2.1. Condition (2.1) in (A1) is equivalent to $\|\phi(0)\| \leq$ $C\|\phi\|_{\mathcal{B}}$, for all $\phi \in \mathcal{B}$.

Example 2.2. The phase space $C_{r} \times L^{p}(g, X)$.

Let $r \geq 0,1 \leq p<\infty$, and let $g:(-\infty,-r) \rightarrow \mathbb{R}$ be a nonnegative measurable function which satisfies the conditions $(g-5),(g-6)$ in the terminology of [21]. Briefly, this means that $g$ is locally integrable, and there exists a nonnegative, locally bounded function $\Lambda$ on $(-\infty, 0]$, such that $g(\xi+\theta) \leq \Lambda(\xi) g(\theta)$, for all $\xi \leq 0$ and $\theta \in(-\infty,-r) \backslash N_{\xi}$, where $N_{\xi} \subseteq(-\infty,-r)$ is a set with Lebesgue measure zero.

The space $C_{r} \times L^{p}(g, X)$ consists of all classes of functions $\varphi$ : $(-\infty, 0] \rightarrow X$ such that $\varphi$ is continuous on $[-r, 0]$, Lebesgue-measurable 
and $g\|\varphi\|^{p}$ on $(-\infty,-r)$. The seminorm in $\|\cdot\|_{\mathcal{B}}$ is defined by

$$
\|\varphi\|_{\mathcal{B}}=\sup _{\theta \in[-r, 0]}\|\varphi(\theta)\|+\left(\int_{-\infty}^{-r} g(\theta)\|\varphi(\theta)\|^{p} d \theta\right)^{1 / p} .
$$

The space $\mathcal{B}=C_{r} \times L^{p}(g, X)$ satisfies axioms (A1), (A2) and (A3). Moreover, for $r=0$ and $p=2$, this space coincides with $C_{0} \times L^{2}(g, X)$, $H=1, M(t)=\Lambda(-t)^{1 / 2}, K(t)=1+\left(\int_{-r}^{0} g(\tau) d \tau\right)^{1 / 2}$, for $t \geq 0$ (see [21, Theorem 1.3.8], for details).

Definition 2.3. Let $\alpha>0$ and $f: \mathbb{R}_{+} \rightarrow X$ be in $L^{1}\left(\mathbb{R}_{+}, X\right)$. Then the Riemann-Liouville integral is given by:

$$
I_{t}^{\alpha} f(t)=\frac{1}{\Gamma(\alpha)} \int_{0}^{t} \frac{f(s)}{(t-s)^{1-\alpha}} d s
$$

where $\Gamma($.$) is the Euler gamma function.$

Definition 2.4 ([34]). The Caputo derivative of order $\alpha$ for a function $f:[0,+\infty) \rightarrow X$ can be written as

$$
\begin{aligned}
D_{t}^{\alpha} f(t) & =\frac{1}{\Gamma(n-\alpha)} \int_{0}^{t} \frac{f^{(n)}(s)}{(t-s)^{\alpha+1-n}} d s \\
& =I^{n-\alpha} f^{(n)}(t), \quad t>0, n-1 \leq \alpha<n .
\end{aligned}
$$

If $0 \leq \alpha<1$, then

$$
D_{t}^{\alpha} f(t)=\frac{1}{\Gamma(1-\alpha)} \int_{0}^{t} \frac{f^{\prime}(s)}{(t-s)^{\alpha}} d s .
$$

Obviously, the Caputo derivative of a constant is equal to zero.

Definition 2.5. A function $f: J \times \mathcal{B} \times X \rightarrow X$ is said to be a Carathéodory function if it satisfies:

(i) for each $t \in J$ the function $f(t, \cdot, \cdot): \mathcal{B} \times X \rightarrow X$ is continuous;

(ii) for each $(v, w) \in \mathcal{B} \times X$, the function $f(\cdot, v, w): J \rightarrow X$ is measurable.

Definition 2.6. Problem (1.1) is said to be controllable on interval $J$ if, for every initial function $\phi \in \mathcal{B}$ and $x_{1} \in X$ there exists a 
control $u \in L^{2}(J, X)$ such that the mild solution $x(\cdot)$ of (1.1) satisfies $x(T)=x_{1}$.

Next we give the concept of a measure of noncompactness [8].

Definition 2.7. Let $B$ be a bounded subset of a seminormed linear space $Y$. Kuratowski's measure of noncompactness of $B$ is defined as $\alpha(B)=\inf \{d>0: B$ has a finite cover by sets of diameter $\leq d\}$.

We need to use the following basic properties of the $\alpha$ measure and Sadovskii's fixed point theorem here (see [36]).

Lemma 2.8. Let $A$ and $B$ be two bounded sets of a Banach space $X$. Then:

(i) If $A \subseteq B$ then $\alpha(A) \leq \alpha(B)$,

(ii) $\alpha(A)=0 \Leftrightarrow \bar{A}$ is compact ( $A$ is relatively compact),

(iii) $\alpha(A+B) \leq \alpha(A)+\alpha(B)$.

Theorem 2.9. (Sadovskii's fixed point theorem). Let $\mathcal{N}$ be a condensing operator on a Banach space $X$, i.e., $\mathcal{N}$ is continuous and takes bounded sets into bounded sets, and $\alpha(\mathcal{N}(D))<\alpha(D)$ for every bounded set $D$ of $X$ with $\alpha(D)>0$. If $\mathcal{N}(S) \subset S$ for a convex, closed and bounded set $S$ of $X$, then $\mathcal{N}$ has a fixed point in $S$.

3. Controllability results. In this section, we prove the main results for controllability of the system (1.1). We give first the definition of the mild solution of the problem.

Definition 3.1. A function $x:(-\infty, T] \rightarrow X$ is said to be a mild solution of (1.1) if $x_{0}=\phi, x_{\rho\left(\tau, x_{\tau}\right)} \in \mathcal{B}$ for every $\tau \in J$ and

$$
\begin{aligned}
x(t)= & -Q(t) \phi(0)+\int_{0}^{t} R(t-s) B u(s) d s \\
& +\int_{0}^{t} \int_{0}^{s} R(t-s) a(s, \tau) f\left(\tau, x_{\rho\left(\tau, x_{\tau}\right)}, x(\tau)\right) d \tau d s, \quad t \in J
\end{aligned}
$$


where

$$
\begin{aligned}
& Q(t)=\int_{0}^{\infty} \xi_{q}(\sigma) S\left(t^{q} \sigma\right) d \sigma \\
& R(t)=q \int_{0}^{\infty} \sigma t^{q-1} \xi_{q}(\sigma) S\left(t^{q} \sigma\right) d \sigma
\end{aligned}
$$

and $\xi_{q}$ is a probability density function defined on $(0, \infty)$ such that

$$
\xi_{q}(\sigma)=\frac{1}{q} \sigma^{-1-1 / q} \varpi_{q}\left(\sigma^{-1 / q}\right) \geq 0,
$$

where

$$
\begin{gathered}
\varpi_{q}(\sigma)=\frac{1}{\pi} \sum_{n=1}^{\infty}(-1)^{n-1} \sigma^{-q n-1} \frac{\Gamma(n q+1)}{n !} \sin (n \pi q), \\
\sigma \in(0, \infty) .
\end{gathered}
$$

Remark 3.2. Note that $\{S(t)\}_{t \geq 0}$ is a uniformly bounded semigroup, i.e., there exists a constant

$$
M>0 \text { such that }\|S(t)\| \leq M \text { for all } t \in[0, T] .
$$

More details on semigroups and their properties can be found in $[9,33]$.

Remark 3.3. According to [29], a direct calculation gives that

$$
\|R(t)\| \leq C_{q, M} t^{q-1}, \quad t>0
$$

where $C_{q, M}=q M / \Gamma(1+q)$.

Set

$$
\mathcal{R}\left(\rho^{-}\right)=\{\rho(s, \varphi):(s, \varphi) \in J \times \mathcal{B}, \rho(s, \varphi) \leq 0\}
$$

We always assume that $\rho: J \times \mathcal{B} \rightarrow(-\infty, T]$ is continuous. Additionally, we introduce following hypothesis:

$\left(H_{\varphi}\right)$ The function $t \rightarrow \varphi_{t}$ is continuous from $\mathcal{R}\left(\rho^{-}\right)$into $\mathcal{B}$, and there exists a continuous and bounded function $L^{\phi}: \mathcal{R}\left(\rho^{-}\right) \rightarrow(0, \infty)$ 
such that

$$
\left\|\phi_{t}\right\|_{\mathcal{B}} \leq L^{\phi}(t)\|\phi\|_{\mathcal{B}} \quad \text { for every } t \in \mathcal{R}\left(\rho^{-}\right) .
$$

Remark 3.4. The condition $\left(H_{\varphi}\right)$, is frequently verified by functions continuous and bounded. For more details, see, for instance, [21].

Remark 3.5. In the rest of this section, $C_{1}^{*}$ and $C_{2}^{*}$ are the constants

$$
C_{1}^{*}=\sup _{t \in J} C_{1}(t) ; \quad C_{2}^{*}=\sup _{t \in J} C_{2}(t) .
$$

Lemma 3.6. ([20]). If $x:(-\infty, T] \rightarrow X$ is a function such that $x_{0}=\phi$, then

$$
\begin{gathered}
\left\|x_{s}\right\|_{\mathcal{B}} \leq\left(C_{2}^{*}+L^{\phi}\right)\|\phi\|_{\mathcal{B}}+C_{1}^{*} \sup \{|y(\theta)| ; \theta \in[0, \max \{0, s\}]\}, \\
s \in \mathcal{R}\left(\rho^{-}\right) \cup J
\end{gathered}
$$

where $L^{\phi}=\sup _{t \in \mathcal{R}\left(\rho^{-}\right)} L^{\phi}(t)$.

Now we introduce the following assumptions:

(H1) $f: J \times \mathcal{B} \times X \rightarrow X$ satisfies the Carathéodory conditions, and there exists a positive function $\mu_{1}(t) \in L^{1}\left(J, \mathbb{R}^{+}\right)$such that

$$
\begin{gathered}
\|f(t, v, w)\| \leq \mu_{1}(t)\left(\|v\|_{\mathcal{B}}+\|w\|_{X}\right), \\
(t, v, w) \in J \times \mathcal{B} \times X .
\end{gathered}
$$

(H2) For each $t \in J, a(t, s)$ is measurable on $[0, t]$ and $a(t)=$ ess $\sup \{|a(t, s)|, 0 \leq s \leq t\}$ is bounded on $J$. The map $t \rightarrow a_{t}$ is continuous from $J$ to $L^{\infty}(J, \mathbb{R})$; here, $a_{t}(s)=a(t, s)$.

(H3) The linear operator $W: L^{2}(J, X) \rightarrow X$ defined by

$$
W u=\int_{0}^{T} R(T-s) B u(s) d s,
$$

has an inverse operator $\widetilde{W}^{-1}$, which takes values in $L^{2}(J, X) /$ ker $W$, and there exist two positive constants $M_{1}$ and $M_{2}$ such that

$$
\|B\|_{L(X)} \leq M_{1}, \quad\left\|\widetilde{W}^{-1}\right\|_{L(X)} \leq M_{2}
$$


(H4) Let

$$
\frac{M_{1} M_{2} a^{*} C_{q, M}^{2} T^{2 q}\left\|\mu_{1}\right\|_{L^{1}}\left(C_{1}^{*}+1\right)}{q^{2}}<1
$$

where $a^{*}=\sup _{t \in J} a(t)$.

\section{Remark 3.7.}

(i) The construction of the bounded inverse operator $\widetilde{W}^{-1}$ in general Banach space is outlined in Remark 3.10.

(ii) When the space $X$ is of finite dimension, condition (H3) is equivalent to the assumption that the Gramian matrix is invertible, or positive definite; see $[\mathbf{7}, \mathbf{4 1}]$.

(iii) In general Banach spaces, condition (H3) has been widely used by many authors; see, for instance, the papers $[\mathbf{2 8 , 4 0 ]}$ and the references therein.

Theorem 3.8. If the hypotheses $\left(H_{\varphi}\right)$ and $(\mathrm{H} 1)-(\mathrm{H} 4)$ are satisfied, and if

$$
\left(C_{1}^{*}+1\right)\left\|\mu_{1}\right\|_{L^{1}}<1
$$

then the problem (1.1) is controllable on the interval $(-\infty, T]$.

Proof. Let $Y=\{u \in C(J, X): u(0)=\phi(0)=0\}$ be endowed with the uniform convergence topology and $N: Y \rightarrow Y$ defined by

$$
\begin{aligned}
N(x)(t)= & -Q(t) \phi(0)+\int_{0}^{t} R(t-s) B u(s) d s \\
& +\int_{0}^{t} \int_{0}^{s} R(t-s) a(s, \tau) f\left(\tau, \bar{x}_{\rho\left(\tau, \bar{x}_{\tau}\right)}, \bar{x}(\tau)\right) d \tau d s, \quad t \in J
\end{aligned}
$$

where $\bar{x}:(-\infty, T] \rightarrow X$ is such that $\bar{x}_{0}=\phi$ and $\bar{x}=x$ on $J$. Let $\bar{\phi}:(-\infty, T] \rightarrow X$ be the extension of $\phi$ to $(-\infty, T]$ such that $\bar{\phi}(\theta)=\phi(0)=0$ on $J$. 
Define the control $u \in L^{2}(J, X)$ by

$$
\begin{aligned}
u(t)= & \widetilde{W}^{-1}\left[x_{1}+Q(t) \phi(0)\right. \\
& \left.-\int_{0}^{T} \int_{0}^{s} R(T-s) a(s, \tau) f\left(\tau, \bar{x}_{\rho\left(\tau, \bar{x}_{\tau}\right)}, \bar{x}(\tau)\right) d \tau d s\right](t) .
\end{aligned}
$$

Choose

$$
r \geq \frac{\frac{M_{1} M_{2} a^{*} C_{q, M}^{2} T^{2 q}\left\|\mu_{1}\right\|_{L^{1}}\left(C_{2}^{*}+L^{\phi}\right)\|\phi\|_{\mathcal{B}}}{q^{2}}}{1-\frac{M_{1} M_{2} a^{*} C_{q, M}^{2} T^{2 q}\left\|\mu_{1}\right\|_{L^{1}}\left(C_{1}^{*}+1\right)}{q^{2}}},
$$

and consider the set

$$
B_{r}=\left\{x \in Y:\|x\|_{\infty} \leq r\right\} .
$$

Clearly, the subset $B_{r}$ is closed, bounded, and convex.

We need the following lemma.

Lemma 3.9. If $x \in B_{r}$, then we have

$$
\left\|\bar{x}_{\rho\left(t, \bar{x}_{t}\right)}\right\|_{\mathcal{B}} \leq\left(C_{2}^{*}+L^{\phi}\right)\|\phi\|_{\mathcal{B}}+C_{1}^{*} r,
$$

and

$$
\begin{aligned}
\|u(s)\| \leq M_{2} & {\left[\left\|x_{1}\right\|+M C\left\|_{\phi}\right\|_{\mathcal{B}}\right.} \\
& \left.+a^{*} C_{q, M} \int_{0}^{T} \int_{0}^{\tau}(t-\tau)^{q-1}\left\|f\left(\iota, \bar{x}_{\rho\left(\iota, \bar{x}_{\iota}\right)}, \bar{x}(\iota)\right)\right\| d \iota d \tau\right] .
\end{aligned}
$$

Proof. Using Lemma 3.6 and equations (3.4) and (3.6), we obtain

$$
\begin{aligned}
\left\|\bar{x}_{\rho\left(t, \bar{x}_{t}\right)}\right\|_{\mathcal{B}} & \leq\left(C_{2}^{*}+L^{\phi}\right)\|\phi\|_{\mathcal{B}}+C_{1}^{*} \sup \{|y(\theta)| ; \theta \in[0, \max \{0, t\}]\} \\
& \leq\left(C_{2}^{*}+L^{\phi}\right)\|\phi\|_{\mathcal{B}}+C_{1}^{*} r .
\end{aligned}
$$

Also, we get

$$
\begin{aligned}
\|u(s)\| \leq & \left\|W^{-1}\right\|\left[\left\|x_{1}\right\|+\|Q(t) \phi(0)\|\right] \\
& +\left\|W^{-1}\right\|\left[\int_{0}^{T} \int_{0}^{\tau}\|R(t-\tau)\|\|a(\tau, \iota)\|\left\|f\left(\iota, \bar{x}_{\rho\left(\iota, \bar{x}_{\iota}\right)}, \bar{x}(\iota)\right)\right\| d \iota d s\right]
\end{aligned}
$$




$$
\begin{aligned}
\leq & M_{2}\left[\left\|x_{1}\right\|+M C\|\phi\|_{\mathcal{B}}\right. \\
& \left.+a^{*} C_{q, M} \int_{0}^{T} \int_{0}^{\tau}(t-\tau)^{q-1}\left\|f\left(\iota, \bar{x}_{\rho\left(\iota, \bar{x}_{\iota}\right)}, \bar{x}(\iota)\right)\right\| d \iota d \tau\right] .
\end{aligned}
$$

The lemma is proved.

Now we decompose $N$ as $N_{1}+N_{2}$ on $B_{r}$, where

$$
\left(N_{1} x\right)(t)=\int_{0}^{t} R(t-s) B u(s) d s, \quad t \in J,
$$

and

$$
\begin{gathered}
\left(N_{2} x\right)(t)=-Q(t) \phi(0)+\int_{0}^{t} \int_{0}^{s} R(t-s) a(s, \tau) f\left(\tau, \bar{x}_{\rho\left(\tau, \bar{x}_{\tau}\right)}, \bar{x}(\tau)\right) d \tau d s, \\
t \in J .
\end{gathered}
$$

Firstly, we show that the operator $N_{1}$ maps $B_{r}$ into itself. Next, we prove that $N_{2}$ is completely continuous. In order to apply Theorem 2.9, we give the proof in several steps.

Step 1. Let $x \in B_{r}$, then show that $N_{1} x \in B_{r}$. For $t \in J$, we have

$$
\begin{aligned}
\left\|\left(N_{1} x\right)(t)\right\| \leq & \int_{0}^{t}\|R(t-s) B u(s)\| d s \\
\leq & M_{1} M_{2} C_{q, M} \\
& \times \int_{0}^{t}(t-s)^{q-1}\left[\left\|x_{1}\right\|+M C\|\phi\|_{\mathcal{B}}+a^{*} C_{q, M}\right. \\
& \left.\times \int_{0}^{T} \int_{0}^{\tau}(t-\tau)^{q-1}\left\|f\left(\iota, \bar{x}_{\rho\left(\iota, \bar{x}_{\iota}\right)}, \bar{x}(\iota)\right)\right\| d \iota d \tau\right] d s \\
\leq & M_{1} M_{2} C_{q, M} \frac{T^{q}}{q}\left[\left\|x_{1}\right\|+M C\left\|_{\phi}\right\|_{\mathcal{B}}\right. \\
& \left.+a^{*} C_{q, M} \frac{T^{q}}{q}\left\|\mu_{1}\right\| L^{1}\left(C_{2}^{*}+L^{\phi}\right)\left\|_{\phi}\right\|_{\mathcal{B}}+\left(C_{1}^{*}+1\right) r\right] \\
\leq & \frac{M_{1} M_{2} C_{q, M} T^{q}}{q}\left[\left\|x_{1}\right\|+M C\left\|_{\phi}\right\|_{\mathcal{B}}\right] \\
& +\frac{M_{1} M_{2} a^{*} C_{q, M}^{2} T^{2 q}\left\|\mu_{1}\right\|_{L^{1}}}{q^{2}}\left[\left(C_{2}^{*}+L^{\phi}\right)\|\phi\|_{\mathcal{B}}+\left(C_{1}^{*}+1\right) r\right] \\
\leq & r .
\end{aligned}
$$


Step 2. $N_{2}$ is continuous. Let $\left\{x^{n}\right\}_{n \in \mathbb{N}}$ be a sequence such that $x^{n} \rightarrow x$ in $B_{r}$ as $n \rightarrow \infty$. Since $f$ satisfies (H1), for almost every $t \in J$, we get

$$
f\left(\tau, \bar{x}_{\rho\left(\tau, \bar{x}_{\tau}^{n}\right)}^{n}, \bar{x}^{n}(\tau)\right) \longrightarrow f\left(\tau, \bar{x}_{\rho\left(\tau, \bar{x}_{\tau}\right)}, \bar{x}(\tau)\right), \quad \text { as } n \rightarrow \infty .
$$

The Lebesgue dominated convergence theorem implies that

$$
\begin{aligned}
\| & \left(N_{2} x^{n}\right)(t)-\left(N_{2} x\right)(t) \| \\
\leq & \int_{0}^{t} \int_{0}^{s}\|R(t-s)\|\|a(s, \tau)\| \| f\left(\tau, \bar{x}_{\rho\left(\tau, \bar{x}_{\tau}^{n}\right)}^{n}, \bar{x}^{n}(\tau)\right) \\
& -f\left(\tau, \bar{x}_{\rho\left(\tau, \bar{x}_{\tau}\right)}, \bar{x}(\tau)\right) \| d \tau d s \\
\leq & a^{*} C_{q, M} \int_{0}^{t} \int_{0}^{s}(t-s)^{q-1} \| f\left(\tau, \bar{x}_{\rho\left(\tau, \bar{x}_{\tau}^{n}\right)}^{n}, \bar{x}^{n}(\tau)\right) \\
& -f\left(\tau, \bar{x}_{\rho\left(\tau, \bar{x}_{\tau}\right)}, \bar{x}(\tau)\right) \| d \tau d s .
\end{aligned}
$$

Hence,

$$
\lim _{n \rightarrow \infty}\left\|\left(N_{2} x^{n}\right)(t)-\left(N_{2} x\right)(t)\right\|=0 .
$$

This means that $N_{2}$ is continuous.

Step 3. We show that $N_{2}\left(B_{r}\right) \subset B_{r}$. For this, we prove by contradiction that there exists a function $x^{r}(\cdot) \in B_{r}$ and $t \in J$ such that $\left\|\left(N_{2} x^{r}\right)(t)\right\|>r$. Thus, from (3.7), we have

$$
\begin{aligned}
r< & \left\|\left(N_{2} x^{r}\right)(t)\right\| \\
\leq & \|-Q(t) \phi(0)\| \\
& +\int_{0}^{t} \int_{0}^{s}\left\|R(t-s) a(s, \tau) f\left(\tau, \bar{x}_{\rho\left(\tau, \bar{x}_{\tau}^{r}\right)}^{r}, \bar{x}^{r}(\tau)\right)\right\| d \tau d s \\
\leq & M C\|\phi\|_{\mathcal{B}}+a^{*} C_{q, M} \\
& \times \int_{0}^{t} \int_{0}^{s}(t-s)^{q-1} \mu_{1}(\tau)\left(\left\|\bar{x}_{\rho\left(\tau, \bar{x}_{\tau}^{r}\right)}^{r}\right\|+\left\|\bar{x}^{r}(\tau)\right\|\right) d \tau d s \\
\leq & M C\|\phi\|_{\mathcal{B}} \\
& +a^{*} C_{q, M}\left(\left(C_{2}^{*}+L^{\phi}\right)\|\phi\|_{\mathcal{B}}+C_{1}^{*} r+r\right) \\
& \times \int_{0}^{t} \int_{0}^{s}(t-s)^{q-1} \mu_{1}(\tau) d \tau d s \\
\leq & M C\|\phi\|_{\mathcal{B}}
\end{aligned}
$$




$$
+\frac{T^{q} a^{*} C_{q, M}}{q}\left(\left(C_{2}^{*}+L^{\phi}\right)\|\phi\|_{\mathcal{B}}+\left(C_{1}^{*}+1\right) r\right)\left\|\mu_{1}\right\|_{L^{1}} .
$$

Dividing on both sides by $r$ and taking the lower limit as $r \rightarrow \infty$, we have

$$
\left(C_{1}^{*}+1\right)\left\|\mu_{1}\right\|_{L^{1}} \geq 1 .
$$

This contradicts condition (3.5). Hence, $N_{2}\left(B_{r}\right) \subset B_{r}$.

Step 4. $N_{2}\left(B_{r}\right)$ is bounded and equicontinuous. By Step 2, it is obvious that $N_{2}\left(B_{r}\right) \subset B_{r}$ is bounded. For the equicontinuity of $N_{2}\left(B_{r}\right)$, set

$$
G\left(\cdot, \bar{x}_{\rho\left(\cdot, \bar{x}_{(\cdot)}\right)}, \bar{x}(\cdot)\right)=\int_{0}^{\cdot} a(\cdot, \tau) f\left(\tau, \bar{x}_{\rho\left(\tau, \bar{x}_{\tau}\right)}, \bar{x}(\tau)\right) d \tau .
$$

Let $0<\tau_{2}<\tau_{1}<T$ and $x \in B_{r}$. Then we can see

$$
\left\|\left(N_{2} x\right)\left(\tau_{1}\right)-\left(N_{2} x\right)\left(\tau_{2}\right)\right\| \leq I_{1}+I_{2}+I_{3},
$$

where

$$
\begin{aligned}
& I_{1}=\left\|Q\left(\tau_{1}\right)-Q\left(\tau_{2}\right)\right\|\|\phi(0)\|, \\
& I_{2}=\left\|\int_{0}^{\tau_{2}}\left[R\left(\tau_{1}-s\right)-R\left(\tau_{2}-s\right)\right] G\left(s, \bar{x}_{\rho\left(s, \bar{x}_{s}\right)}, \bar{x}(s)\right) d s\right\|, \\
& I_{3}=\int_{\tau_{2}}^{\tau_{1}}\left\|R\left(\tau_{1}-s\right)\right\|\left\|G\left(s, \bar{x}_{\rho\left(s, \bar{x}_{s}\right)}, \bar{x}(s)\right)\right\| d s .
\end{aligned}
$$

The continuity of $S(t)$ in the uniform operator topology follows for $t>0$ such that $I_{1}$ tends to zero, as $\tau_{2} \rightarrow \tau_{1}$.

In view of (3.2), we have

$$
\begin{aligned}
I_{2} \leq & \| \int_{0}^{\tau_{2}}\left[q \int_{0}^{\infty} \sigma\left(\tau_{1}-s\right)^{q-1} \xi_{q}(\sigma) S\left(\left(\tau_{1}-s\right)^{q} \sigma\right) \| d \sigma\right. \\
& \left.-q \int_{0}^{\infty} \sigma\left(\tau_{2}-s\right)^{q-1} \xi_{q}(\sigma) S\left(\left(\tau_{2}-s\right)^{q} \sigma\right) d \sigma\right] \\
& \times G\left(s, \bar{x}_{\rho\left(s, \bar{x}_{s}\right)}, \bar{x}(s)\right) d s \| \\
\leq & q \int_{0}^{\tau_{2}} \int_{0}^{\infty} \sigma \|\left[\left(\tau_{1}-s\right)^{q-1}-\left(\tau_{2}-s\right)^{q-1}\right] \xi_{q}(\sigma) S\left(\left(\tau_{1}-s\right)^{q} \sigma\right) \\
\times & G\left(s, \bar{x}_{\rho\left(s, \bar{x}_{s}\right)}, \bar{x}(s)\right) \| d \sigma d s \\
& +q \int_{0}^{\tau_{2}} \int_{0}^{\infty} \sigma\left(\tau_{2}-s\right)^{q-1} \xi_{q}(\sigma) \|
\end{aligned}
$$




$$
\begin{aligned}
\times & S\left(\left(\tau_{1}-s\right)^{q} \sigma\right)-S\left(\left(\tau_{2}-s\right)^{q} \sigma\right) \| \\
\times & \left\|G\left(s, \bar{x}_{\rho\left(s, \bar{x}_{s}\right)}, \bar{x}(s)\right)\right\| d \sigma d s \\
\leq & C_{q, M} \int_{0}^{\tau_{2}}\left|\left(\tau_{1}-s\right)^{q-1}-\left(\tau_{2}-s\right)^{q-1}\right| \\
\times & \left\|G\left(s, \bar{x}_{\rho\left(s, \bar{x}_{s}\right)}, \bar{x}(s)\right)\right\| d s \\
& +q \int_{0}^{\tau_{2}} \int_{0}^{\infty} \sigma\left(\tau_{2}-s\right)^{q-1} \xi_{q}(\sigma)\left\|S\left(\left(\tau_{1}-s\right)^{q} \sigma\right)-S\left(\left(\tau_{2}-s\right)^{q} \sigma\right)\right\| \\
\times & \left\|G\left(s, \bar{x}_{\rho\left(s, \bar{x}_{s}\right)}, \bar{x}(s)\right)\right\| d \sigma d s \\
\leq & a^{*}\left\|\mu_{1}\right\|_{L^{1}}\left[\left(C_{2}^{*}+L^{\phi}\right)\|\phi\|_{\mathcal{B}}+\left(C_{1}^{*}+1\right) r\right] \\
\times & {\left[C_{q, M} \int_{0}^{\tau_{2}}\left|\left(\tau_{1}-s\right)^{q-1}-\left(\tau_{2}-s\right)^{q-1}\right| d s\right.} \\
& +q \int_{0}^{\tau_{2}} \int_{0}^{\infty} \sigma\left(\tau_{2}-s\right)^{q-1} \xi_{q}(\sigma) \\
\times & \left.\left\|S\left(\left(\tau_{1}-s\right)^{q} \sigma\right)-S\left(\left(\tau_{2}-s\right)^{q} \sigma\right)\right\| d \sigma d s\right] .
\end{aligned}
$$

Clearly, the first term on the right-hand side of the above inequality tends to 0 as $\tau_{2} \rightarrow \tau_{1}$. The second term on the right-hand side of the above inequality tends to 0 as $\tau_{2} \rightarrow \tau_{1}$ as a consequence of the continuity of $S(t)$ in the uniform operator topology for $t>0$. For $I_{3}$, we have

$$
\begin{aligned}
I_{3} & \leq C_{q, M} \int_{\tau_{2}}^{\tau_{1}}\left(\tau_{1}-s\right)^{q-1}\left\|G\left(s, \bar{x}_{\rho\left(s, \bar{x}_{s}\right)}, \bar{x}(s)\right)\right\| d s \\
\leq & a^{*} C_{q, M}\left[\left(C_{2}^{*}+L^{\phi}\right)\|\phi\|_{\mathcal{B}}+\left(C_{1}^{*}+1\right) r\right] \\
& \times \int_{\tau_{2}}^{\tau_{1}}\left(\tau_{1}-s\right)^{q-1} d s \\
& \stackrel{\longrightarrow}{\longrightarrow}, \text { as } \tau_{2} \rightarrow \tau_{1} .
\end{aligned}
$$

From the above, it is clear that $N_{2}\left(B_{r}\right)$ is equicontinuous.

Finally, combining Step 2-Step 4 together with Ascoli's theorem, we conclude that the operator $N_{2}$ is compact. In fact, by Step 1-Step 4 
and Lemma 2.8, one can conclude that $N=N_{1}+N_{2}$ is continuous and takes bounded sets into bounded sets.

Meanwhile, it is easy to see $\alpha\left(N_{2}\left(B_{r}\right)\right)=0$ since $N_{2}\left(B_{r}\right)$ is relatively compact. It follows from $N_{1}\left(B_{r}\right) \subseteq B_{r}$ and $\alpha\left(N_{2}\left(B_{r}\right)\right)=0$ that $\alpha\left(N\left(B_{r}\right)\right) \leq \alpha\left(N_{1}\left(B_{r}\right)\right)+\alpha\left(N_{2}\left(B_{r}\right)\right) \leq \alpha\left(B_{r}\right)$ for every bounded set $B_{r}$ of $X$ with $\alpha\left(B_{r}\right)>0$.

Since $N\left(B_{r}\right) \subset B_{r}$ for a convex, closed and bounded set $B_{r}$ of $Y$ using Theorem 2.9, we conclude that $N$ has a fixed point $x \in B_{r}$. Hence, $N$ has a fixed point which is a mild solution to the problem (1.1) satisfying $x(T)=x_{1}$. Thus, system (1.1) is controllable on $(-\infty, T]$.

Remark 3.10. (see also [35]). Construction of $\widetilde{W}^{-1}$. Let $E=$ $L^{2}(J, U) / \operatorname{ker} W$. Since ker $W$ is closed, $E$ is a Banach space under the norm

$$
\|\bar{u}\|_{E}=\inf _{u \in \bar{u}}\|u\|_{L^{2}(J, U)}=\inf _{W \hat{u}=0}\|u+\widehat{u}\|_{L^{2}(J, U)},
$$

where $\bar{u}$ are the equivalence classes of $u$.

Define $\widetilde{W}: E \rightarrow X$ by

$$
\widetilde{W} \bar{u}=W u, \quad u \in \bar{u} .
$$

Now $\widetilde{W}$ is one-to-one and

$$
\|\widetilde{W} \bar{u}\|_{X} \leq\|W\|\|\bar{u}\|_{E}
$$

We claim that $V=$ Range $W$ is a Banach space with the norm

$$
\|v\|_{V}=\left\|\widetilde{W}^{-1} v\right\|_{E}
$$

This norm is equivalent to the graph norm on $D\left(\widetilde{W}^{-1}\right)=$ Range $W, \widetilde{W}$ is bounded and since $D(\widetilde{W})=E$ is closed, $\widetilde{W}^{-1}$ is closed and so the above norm makes Range $W=V$, a Banach space.

Moreover,

$$
\begin{aligned}
\|W u\|_{V} & =\left\|\widetilde{W}^{-1} W u\right\|_{E}=\left\|\widetilde{W}^{-1} \widetilde{W} \bar{u}\right\|_{E} \\
& =\|\bar{u}\|=\inf _{u \in \bar{u}}\|u\| \leq\|u\|,
\end{aligned}
$$

so

$$
W \in \mathcal{L}\left(L^{2}(J, U), V\right) .
$$


Since $L^{2}(J, U)$ is reflexive and $\operatorname{ker} W$ is weakly closed, the infimum is actually attained. For any $v \in V$, we can therefore choose a control $u \in L^{2}(J, U)$ such that $u=\widetilde{W}^{-1} v$.

4. An example. To apply our abstract results, we consider the fractional integrodifferential equation with state dependent delay of the form

$$
\begin{aligned}
\frac{\partial^{q}}{\partial t^{q}} v(t, \zeta)= & \frac{\partial^{2}}{\partial \zeta^{2}} v(t, \zeta)+\omega \mu(t, \zeta) \\
& +\int_{0}^{t}(t-s)^{2} \int_{-\infty}^{s} \gamma(\tau-s) v\left(\tau-\rho_{1}(s) \rho_{2}(|v(s, \zeta)|), \zeta\right) d \tau d s \\
& +\int_{0}^{t}(t-s)^{2} \sin |v(s, \zeta)| d s, \quad t \in[0, T], \zeta \in[0, \pi], \\
(4.1) \quad t \in[0, T], & v(t, \pi)=0, \quad t \in(-\infty, 0], \zeta \in[0, \pi],
\end{aligned}
$$

where $0<q<1, \omega>0, \mu:[0, T] \times[0, \pi] \rightarrow[0, \pi], \rho_{i}:[0,+\infty) \rightarrow$ $[0,+\infty), i=1,2$, are continuous functions, and $\partial^{q} / \partial t^{q}:=D_{t}^{\alpha}$.

Set $X=L^{2}([0, \pi])$, and define $A$ by

$$
\begin{gathered}
D(A)=\left\{u \in X: u^{\prime \prime} \in X, u(0)=u(\pi)=0\right\}, \\
A u=u^{\prime \prime} .
\end{gathered}
$$

It is well known that $A$ is the infinitesimal generator of an analytic semigroup $(S(t))_{t \geq 0}$ on $X$. Furthermore, $A$ has a discrete spectrum with eigenvalues of the form $-n^{2}, n \in \mathbb{N}$, and the corresponding normalized eigenfunctions are given by

$$
u_{n}(x)=\sqrt{\frac{2}{\pi}} \sin (n x) .
$$

In addition, $\left\{u_{n}: n \in \mathbb{N}\right\}$ is an orthogonal basis for $X$,

$$
S(t) u=\sum_{n=1}^{\infty} e^{-n^{2} t}\left(u, u_{n}\right) u_{n}, \quad \text { for all } u \in X \text { and every } t \geq 0 .
$$

From these expressions, it follows that $(S(t))_{t \geq 0}$ is a uniformly bounded compact semigroup. For the phase space, we choose $\mathcal{B}=C_{0} \times L^{2}(g, X)$, see Example 2.2 for details. 
For $t \in[0, T]$ and $\zeta \in[0, \pi]$, we set

$$
\begin{aligned}
x(t)(\zeta) & =v(t, \zeta), \\
a(t, s) & =(t-s)^{2}, \\
f(t, \varphi, x(t))(\zeta) & =\int_{-\infty}^{0} \gamma(\tau) \varphi(\tau, \zeta) d \tau+\sin |x(t)(\zeta)| \\
\rho(t, \varphi) & =\rho_{1}(t) \rho_{2}(\|\varphi(0)\|) \\
B u(t)(\zeta)=\omega \mu(t, \zeta) &
\end{aligned}
$$

Under the above conditions, we can represent the system (4.1) in the abstract form (1.1). Assume that the operator $W: L^{2}(J, X) \rightarrow X$ defined by

$$
W u(\cdot)=\int_{0}^{T} R(T-s) \omega \mu(s, \cdot) d s,
$$

has a bounded invertible operator $W^{-1}$ in $L^{2}(J, X) / \operatorname{ker} W$.

The following result is a direct consequence of Theorem 3.8.

Proposition 4.1. Let $\varphi \in \mathcal{B}$ be such that $\left(H_{\varphi}\right)$ holds, and assume that the above conditions are fulfilled. Then system (4.1) is controllable on $(-\infty, T]$.

\section{REFERENCES}

1. N. Abada, M. Benchohra and H. Hammouche, Existence and controllability results for nondensely defined impulsive semilinear functional differential inclusions, J. Differ. Equat. 246 (2009), 3834-3863.

2. R. Agarwal, B. de Andrade, and G. Siracusa, On fractional integro-differential equations with state-dependent delay, Comp. Math. Appl. 62 (2011), 1143-1149.

3. R.P. Agarwal, B. de Andrade and C. Cuevas, On type of periodicity and ergodicity to a class of fractional order differential equations, Adv. Diff. Equat. 2010 (2010), article ID 179750.

4. M.M. Arjunan and V. Kavitha, Controllability of impulsive fractional evolution integrodifferential equations in Banach spaces, J. KSIAM 15 (2011), 177-190.

5. K. Balachandran and J.P. Dauer, Controllability of nonlinear systems in Banach spaces: A survey, J. Optim. Th. Appl. 115 (2002), 7-28.

6. K. Balachandran and J.Y. Park, Controllability of fractional integrodifferential systems in Banach spaces, Nonlin. Anal. Hybr. Syst. 3 (2009), 363-367.

7. K. Balachandran, J.Y. Park and J.J. Trujillo, Controllability of nonlinear fractional dynamical systems, Nonlin. Anal. 75 (2012), 1919-1926. 
8. J. Banaś and K. Goebel, Measures of noncompactness in Banach spaces, Lect. Notes Pure Appl. Math., Marcel Dekker, New York, 1980.

9. M. Bartha, Periodic solutions for differential equations with state-dependent delay and positive feedback, Nonlin. Anal. TMA 53 (2003), 839-857.

10. M. Benchohra, L. Góriewicz, S.K. Ntouyas and A. Ouahab, Controllability results for nondensely defined semilinear functional differential equations, $\mathrm{Z}$. Anal. Anwend. 25 (2006), 311-325.

11. M. Benchohra, J. Henderson, S.K. Ntouyas and A. Ouahab, Existence results for fractional functional differential inclusions with infinite delay and application to control theory, Fract. Calc. Appl. Anal. 11 (2008), 35-56.

12. M. Benchohra and S. Litimein, A global uniqueness result for abstract integral equations of Volterra type in Banach spaces, Comm. Appl. Nonlin. Anal. 18 (2011), 37-44.

13. __ Fractional integro-differential equations with state-dependent delay on an unbounded domain, Afr. Diasp. J. Math. 12 (2011), 13-25.

14. M. Benchohra, S. Litimein, J.J. Trujillo and M.P. Velasco, Abstract fractional integro-differential equations with state-dependent delay, Int. J. Evol. Equat. 6 (2012), 25-38.

15. M. Benchohra and A. Ouahab, Controllability results for functional semilinear differential inclusions in Fréchet spaces, Nonlinear Anal. 61 (2005), 405-423.

16. Y.K. Chang and D.N. Chalishajar, Controllability of mixed VolterraFredholm-type integro-differential inclusions in Banach spaces, J. Franklin Inst. 345 (2008), 499-507.

17. Y.Q. Chen, H.S. Ahu and D. Xue, Robust controllability of interval fractional order linear time invariant systems, Signal Proces. 86 (2006), 2794-2802.

18. J.P.C. dos Santos, C. Cuevas and B. de Andrade, Existence results for a fractional equation with state-dependent delay, Adv. Differ. Equat. 2011 (2011), article ID 642013.

19. J.K. Hale and J. Kato, Phase space for retarded equations with infinite delay, Funk. Ekvac. 21 (1978), 11-41.

20. E. Hernández, A. Prokopczyk and L. Ladeira, A note on partial functional differential equations with state-dependent delay, Nonlinear Anal. RWA 7 (2006), $510-519$.

21. Y. Hino, S. Murakami and T. Naito, Functional differential equations with unbounded delay, Springer-Verlag, Berlin, 1991.

22. V. Kavitha and M.M. Arjunan, Controllability of impulsive quasi-linear fractional mixed volterra-fredholm-type integrodifferential equations in Banach spaces, J. Nonlin. Sci. Appl. 4 (2011), 152-169.

23. V. Kavitha, P-Z. Wang and R. Murugesu, Existence results for neutral functional fractional differential equations with state dependent-delay, Malaya J. Math. 1 (2012), 50-61.

24. S. Kumar and N. Sukavanam, Approximate controllability of fractional order neutral control systems with delay, Int. J. Nonlin. Sci. 13 (2012), 454-462. 
25. J. Liang, J.H. Liu and T.-J. Xiao, Nonlocal problems for integrodifferential equations, Dynam. Cont. Disc. Impul. Syst. 15 (2008), 815-824.

26. J. Liang and T.-J. Xiao, Semilinear integrodifferential equations with nonlocal initial conditions, Comp. Math. Appl. 47 (2004), 863-875.

27. J. Liang, T.-J. Xiao and J. van Casteren, A note on semilinear abstract functional differential and integrodifferential equations with infinite delay, Appl. Math. Lett. 17 (2004), 473-477.

28. Y. Liu, and D. O'Regan, Controllability of impulsive functional differential systems with nonlocal conditions, Electr. J. Differ. Equat. 194 (2013).

29. F. Mainardi, P. Paradisi and R. Gorenflo, Probability distributions generated by fractional diffusion equations, in Econophysics: An emerging science, J. Kertesz and I. Kondor, eds., Kluwer Academic Publishers, Dordrecht, The Netherlands, 2000 .

30. M. Matar, Controllability of fractional semilinear mixed Volterra-Fredholm integrodifferential equations with nonlocal conditions, Int. J. Math. Anal. 4 (2010), 1105-1116.

31. D. Matignon and B. D'Andréa-Novel, Some results on controllability and observability of finite-dimensional fractional differential systems, in Proc. IMACS, IEEE SMC Conference, Lille, France, 1996, 952-956.

32. G.M. Mophou and G.M. N'Guérékata, Existence of mild solution for some fractional differential equations with nonlocal conditions, Semigroup Forum $\mathbf{7 9}$ (2009), 315-322.

33. A. Pazy, Semigroups of linear operators and applications to partial differential equations, Springer-Verlag, New York, 1983.

34. I. Podlubny, Fractional differential equations, Academic press, New York, 1993.

35. M.D. Quinn and N. Carmichael, An approach to nonlinear control problems using the fixed point methods, degree theory and pseudo-inverses, Numer. Funct. Anal. Optim. 7 (1984)-(1985), 197-219.

36. B. Sadovskii, On a fixed point principle, Funct. Anal. Appl. 2 (1967), 151153.

37. R. Sakthivel, R. Yong and N.I. Mahmudov, On the approximate controllability of semilinear fractional differential systems, Comp. Math. Appl. 62 (2011), 1451-1459.

38. A.B. Shamardan and M.R.A. Moubarak, Controllability and observability for fractional control systems, J. Fract. Calc. 15 (1999), 25-34.

39. Z. Tai and X. Wang, Controllability of fractional-order impulsive neutral functional infinite delay integrodifferential systems in Banach spaces, Appl. Math. Lett. 22 (2009), 1760-1765.

40. J. Wang, X. Li and W. Wei, On controllability for fractional differential inclusions in Banach spaces, Opuscula Math. 32 (2012), 341-356.

41. J. Wei, The controllability of fractional control systems with control delay, Comp. Math. Appl. 64 (2012), 3153-3159. 
42. T.-J. Xiao and J. Liang, Blow-up and global existence of solutions to integral equations with infinite delay in Banach spaces, Nonlin. Anal. Theor. Meth. Appl. 71 (2009), 1442-1447.

Laboratory of Mathematics, University of Sidi Bel-Abbès, P.O. Box 89, 22000, Sidi Bel-AbBès, Algeria

Email address: aissani_k@yahoo.fr

Laboratory of Mathematics, University of Sidi Bel-Abbès, P.O. Box 89, 22000, Sidi Bel-Abbès, Algeria and Department of Mathematics, King AbDulaziz University, P.O. Box 80203, JedDAH 21589, SAudi Arabia

Email address: benchohra@yahoo.com 